\title{
Double-stranded regions are essential design components of potent inhibitors of RISC function
}

\author{
ANNALEEN VERMEULEN, BARBARA ROBERTSON, ANDREW B. DALBY, WILLIAM S. MARSHALL, \\ JON KARPILOW, DEVIN LEAKE, ANASTASIA KHVOROVA, and SCOTT BASKERVILLE \\ Thermo Fisher Scientific, Dharmacon Products, Lafayette, Colorado 80026, USA
}

\begin{abstract}
While microRNAs (miRNAs) are recognized as playing a critical role in regulating eukaryotic gene expression, both the mechanism by which these small, noncoding RNAs function and the genes they target remain elusive. Previous studies have shown that short, single-stranded 2'-O-methyl-modified oligonucleotides that are complementary to mature microRNA sequences can interact with the miRNA-RISC nucleoprotein complex and weakly inhibit miRNA function. Here we report the identification of secondary structural elements that enhance the potency of these molecules. Incorporation of highly structured, double-stranded flanking regions around the reverse complement core significantly increases inhibitor function and allows for multi-miRNA inhibition at subnanomolar concentrations. The improved functionality of these double-stranded miRNA inhibitors may provide insights into the miRNA mechanism by suggesting the possible importance of such structures in or near endogenous miRNA target sites.
\end{abstract}

Keywords: inhibitor; microRNA; RISC; double stranded; hairpin; RNA; 2'-O-methyl

\section{INTRODUCTION}

MicroRNAs (miRNAs) are a class of short $\sim 22$ nucleotide (nt), noncoding RNAs that modulate gene expression at the post-transcriptional level. These small RNAs are part of a larger ribonucleoprotein complex, the RNA-induced silencing complex (RISC) (Kim 2005; Gregory et al. 2006), which modulates gene expression by two broad classes of translational repression. In cases of complete or near-complete complementarity between the miRNA and the target sequence, the miRNA-loaded RISC (miRISC) induces transcript cleavage that results in rapid and extensive reduction of mRNA levels. Although this mechanism has been observed in animals (Yekta et al. 2004), in general, mammalian miRNAs have limited levels of complementarity with target sequences and instead act by localizing miRNP/mRNA complexes in or near cytoplasmic processing bodies (P-bodies) (Liu et al. 2005; Sen and Blau 2005). This latter process leads to inhibition of translation initiation (Pillai et al. 2005) and/or rapid deadenylation (Giraldez et al. 2006) of the target transcript.

Reprint requests to: Annaleen Vermeulen, Thermo Fisher Scientific, Dharmacon Products, 2650 Crescent Drive, Suite \#100, Lafayette, CO 80026, USA; e-mail: annaleen.vermeulen@thermofisher.com; fax: (303) 604-9680.

Article published online ahead of print. Article and publication date are at http://www.rnajournal.org/cgi/doi/10.1261/rna.448107.
The diverse nature of miRNA target sites is reflected in the results of both experimentally validated and computationally based miRNA target site predictions, which suggest that, on average, each miRNA targets hundreds of genes (Rhoades et al. 2002; Enright et al. 2003; Bino et al. 2004; Kiriakidou et al. 2004; Lewis et al. 2005; Lim et al. 2005; Sethupathy et al. 2006). One approach for deconstructing this complex miRNA-target network is to disrupt miRNA function. Current biochemically based tools for the sequence-specific inhibition of miRNA function are limited to single-stranded oligonucleotide-based miRNA inhibitors. These molecules are fully $2^{\prime}$-O-methylated reverse complements of the mature miRNA sequences (Hutvagner et al. 2004; Meister et al. 2004) and are believed to act as noncleavable substrates (antisense) of RISC. The effects of alternative chemical modification patterns (including locked nucleic acids (LNA), phosphorothioate and 2'methoxyethyl modifications (Krutzfeldt et al. 2005; Davis et al. 2006; Orom et al. 2006), have also been investigated; to date, none of these alterations have shown significant improvements over the original designs. In contrast, slight improvement in inhibitor potency has been observed by increasing the length of the inhibitor (Hutvagner et al. 2004). Still, it remains unclear if there is a specific combination of sequences, structures, and/or chemical modifications that can significantly increase the potency of antisensebased miRNA inhibitors. 
Using a robust reporter system for monitoring endogenous miRNA function, we have investigated the contributions that inhibitor length, symmetry, sequence, chemical modifications, and secondary structure play in inhibitor function. Our findings show that the length and composition of sequences surrounding the reverse complement (RC or antisense) have considerable effects on overall inhibitor potency. Unexpectedly, the most potent inhibitors contained secondary structural elements that flanked the antisense core. Molecules having optimized secondary structures are highly potent and specifically block RISC activity in cell lines for extended periods of time, thus demonstrating that structures surrounding or adjacent to the RC inhibitor sequence are major determinants of inhibitor potency.

\section{RESULTS}

\section{Development of a sensitive microRNA detection system}

To test the functionality of inhibitor variants, a sensitive, robust system for detecting endogenous miRNA expression was needed. To achieve this, an assay system was developed by adapting a vector originally designed for siRNA validation (psiCHECK-2; Promega). The psiCHECK-2 reporter contains modified versions of both Renilla renifomis (sea pansy) luciferase ( $h R l u c$ ), and Photinus pyralis (firefly) luciferase $(h l u c+$ ) on a single plasmid (Supplemental Fig. 1). To optimize psiCHECK-2 for miRNA detection, three separate vector designs having different miRNA recognition elements (MRES) for a highly expressed miRNA (miR-21) (Lim et al. 2003) were constructed. The first design contains a single perfectly complementary "cleavage-based" MRE inserted into the 3' UTR of the hRluc gene. The second and third vector designs contain one or three "attenuationbased" MREs, which are partially complementary to the mature miR-21, inserted in the $3^{\prime}$ UTR of the hRluc gene (Supplemental Table 1A; Supplemental Fig. 1A). Specifically, the two attenuation-based MREs contain four extra nucleotides (internal loop) that have previously been shown to result in translational attenuation (Kiriakidou et al. 2004). Endogenous miRNA function is monitored by measuring the relative expression levels of target luciferase (Renilla, Rluc) and control luciferase (Photinus, Fluc).

When the vectors are introduced into cells, the ratio of Rluc to Fluc expression is reduced for both the "cleavage" and "attenuation" (att1, att3) vector designs relative to psiCHECK-2 control (normalized values $<1$, Fig. 1A; inhibitor [-]). Normalized Rluc/Fluc values are lowest with the cleavage vector, demonstrating that assays based on cleavage are more sensitive and have a greater dynamic range than comparative attenuation assays.

To confirm the mechanism underlying the observed changes in Rluc/Fluc protein expression for each vector,

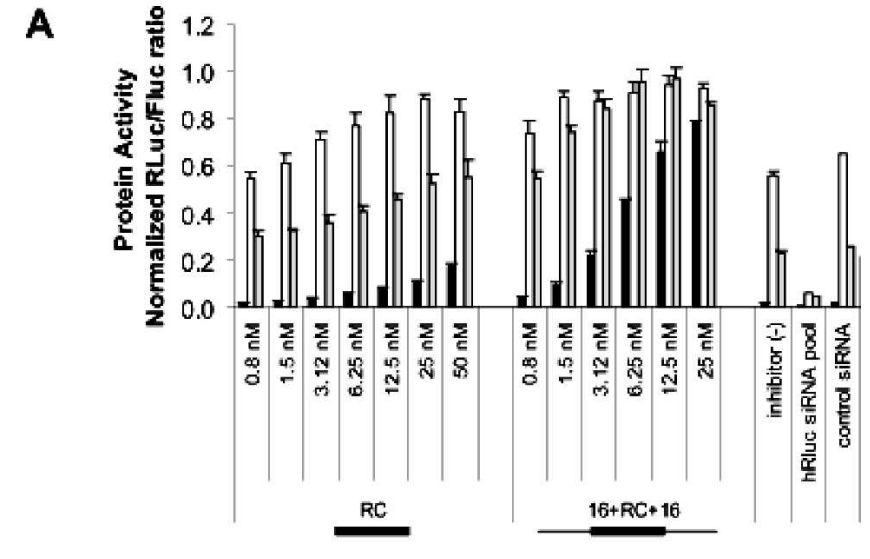

B

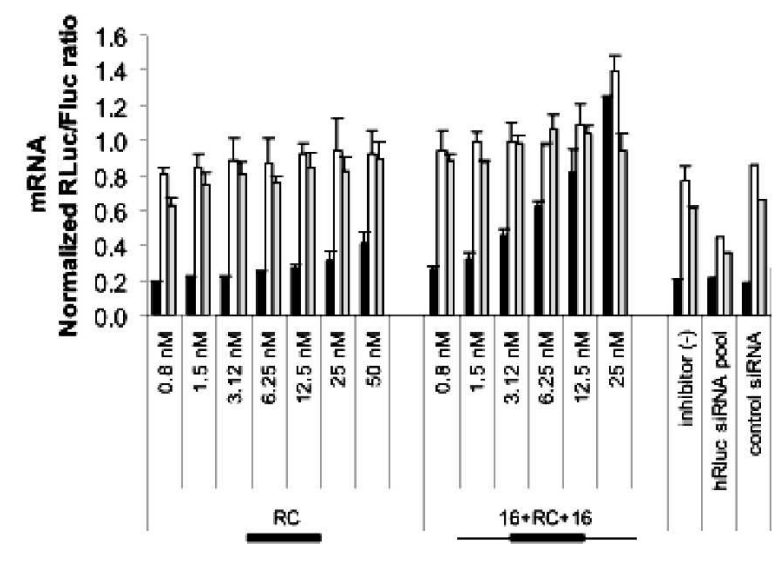

FIGURE 1. MicroRNA inhibitors are effective at repressing both cleavage and translational attenuation pathways. The effect of inhibition of miR-21 was determined using three types of microRNA assays ( $24 \mathrm{~h}$ post-transfection). Black bars indicate cleavage assay; white and gray bars indicate attenuation assays with constructs containing one and three attenuation sites, respectively. Inhibition was determined at the protein level $(A)$ as detected by luciferase activity and at the mRNA level $(B)$ as detected by branched-DNA assay of luciferase. A dose curve of two inhibitor designs, reverse complement to mature (RC) and a longer inhibitor reverse complement to pri-miRNA (16+ $\mathrm{RC}+16$ ) were tested. Controls include no inhibitor (inhibitor [-]), siRNA pool against hRluc gene and negative control siRNA (targeting the nonplasmid protein, PPIB).

luciferase protein and mRNA levels were monitored for endogenous miRNA knockdown and siRNA-mediated knockdown. Delivery of siRNA directed against the open reading frame of Rluc shows protein and mRNA changes that are consistent with the siRNA-directed mRNA cleavage mechanism (Fig. 1A,B; hRluc siRNA pool). Similarly, transfection of the cleavage-based vector alone showed a reduction in both protein and mRNA levels (Fig. 1A,B; inhibitor $[-]$ ), suggesting that in cases where the target site has complete complementarity to the endogenous miRNA (miR-21), the mechanism of action is similar to that of an siRNA (Fig. 1A,B; hRluc siRNA pool). In contrast, while the two attenuation-based vectors show reductions in protein levels in response to endogenous miRNA (Fig. 1A, 
inhibitor [-]), the changes in mRNA levels are distinctly less than those observed in response to exogenously delivered siRNA (Fig. 1B; inhibitor [-], hRluc siRNA pool) indicating that the attenuation-based vectors operate through a translational attenuation-based mechanism.

To (1) identify which vector system was the best to study and optimize miRNA inhibitor design, and (2) test whether inhibitors affected both the cleavage- and the attenuationbased pathways, endogenous miRNA function was tested in the presence or absence of inhibitor molecules designed for each system (Fig. 1A,B). Two fully 2'-O-methylated inhibitor designs were tested: a short (21-nt) reverse complement to the mature miR-21 (RC or antisense core) and a longer molecule that contained the $\mathrm{RC}$ core plus $5^{\prime}$ and $3^{\prime}$ flanking regions that were complementary to sequences bordering the RC in the primary miRNA transcript (primiR, $16+R C+16$ ). Relative to untreated cells, cotransfection of the appropriate reporter construct with each inhibitor led to increases in normalized Rluc/Fluc values in both the attenuation- and cleavage-based systems. The longer reverse complement $(16+\mathrm{RC}+16)$ inhibitor was found to be significantly more potent than the shorter antisense-based inhibitor (Fig. 1A,B), while the control noncognate reporter constructs showed no response to endogenous miRNA levels or to inhibitor treatment (data not shown). In addition, cleavage-based reporters were found to be more sensitive to the presence of inhibitors than either of the vectors that contained $3^{\prime}$-UTR attenuation sites. Overall, these results confirm that the reporter systems described here are responsive to both mechanisms of RISC-mediated silencing. Furthermore, as cleavagebased assays showed heightened sensitivity, all subsequent studies designed to investigate inhibitor design employed cleavage-based reporter constructs to study two moderately to highly expressed miRNAs, let-7c, and miR-21.

\section{Inhibitor length improves inhibitor potency}

To test whether sequences flanking the $\mathrm{RC}$ core could alter the potency of miRNA inhibitors, 2'-O-methyl-modified inhibitor oligonucleotides of varying lengths were synthesized and assayed for the ability to inhibit let- $7 \mathrm{c}$ and miR21 function. Inhibitor sets ranged in length from $22 \mathrm{nt}$ to $54 \mathrm{nt}$, and were designed to contain a constant antisense (RC) core flanked by $5^{\prime}$ and $3^{\prime}$ sequences that were (1) equal in length, and (2) complementary to regions adjacent to the mature miRNA sequence in the target (let-7c or miR-21) pri-miRNA (Fig. 2A).

The results of these studies are presented in Figure 2, B and $\mathrm{C}$, and demonstrate that the inhibitory function of $2^{\prime}$-O-methyl-modified oligonucleotides is strongly dependent upon length. While molecules having symmetrical flanking region lengths of 2, 4, 6, and 8 nt exhibited similar levels of inhibition to simple (21-nt) reverse complement cores, larger molecules having symmetrical flanking regions of $>10$-nt inhibited target miRNAs to a much greater extent. The level of inhibition resulting from increased length peaked at $\sim 52 \mathrm{nt}$ (i.e., 12-14-nt symmetrical flanks). Although changing the location of the antisense core within a 54-nt inhibitor had an effect on inhibitor potency, no consistent trend in overall function was observed (Supplemental Fig. 2). Further studies of long (54-nt) inhibitors containing an assortment of chemical modifications (e.g., 2'-O-methyl, 2'-fluoro, phosphorothioate, or a combination of these modifications) failed to identify any pattern of modifications that significantly enhanced function, and in some cases, identified modifications that greatly reduced the ability to inhibit RISC function (Supplemental Fig. 3). Similar observations have been made by Davis et al. (2006), who tested a selection of modifications in conjunction with short (RC) inhibitors.

\section{Flanking region sequence can affect overall inhibitor function}

To test the effects of flanking sequence composition on inhibitor activity, the flanking sequences employed above were replaced with arbitrary sequences having a mixture of A, G, C, and U (Fig. 3A; 16A + RC + 16A). A side-by-side comparison between inhibitors with complementary $(16+$ $\mathrm{RC}+16)$ or arbitrary $(16 \mathrm{~A}+\mathrm{RC}+16 \mathrm{~A})$ flanks identified a slight but measurable reduction in inhibition by molecules with arbitrary flanking sequences (Fig. 3B), suggesting that elements (sequences or structures) outside the antisense core could influence potency of inhibitors. To further investigate this observation, let-7c and miR-21 inhibitors containing a polypyrimidine $(\mathrm{Y})$ stretch in the $5^{\prime}$ and $3^{\prime}$ flanking sequences $(16 \mathrm{Y}+\mathrm{RC}+16 \mathrm{Y}$; Fig. $3 \mathrm{~A})$ were tested in the dual luciferase reporter assay. Oligonucleotides with polypyrimidine stretches are generally considered to be less structured than sequences having an equal distribution of purines and pyrimidines. As shown in Figure 3B, inhibitors with polypyrimidine flanks were found to be less potent than molecules having flanks that were (1) complementary to the pri-miRNA $(16+\mathrm{RC}+16)$, or (2) arbitrary $(16 \mathrm{~A}+$ $\mathrm{RC}+16 \mathrm{~A})$. The relative absence of function by molecules with polypyrimidine flanks further supports the notion that the structure of regions adjacent to the RC core could play a role in the extent of RISC inhibition.

\section{Incorporation of secondary structure improves inhibitor function}

To further test the contributions of flanking region secondary structure to inhibitor function, the $\mathrm{RC}$ regions of let-7c and miR-21 inhibitors were flanked with sequences capable of forming hairpin structures. In each case, hairpins (hp) were generated from either arbitrary $(8 \mathrm{Ahp}+\mathrm{RC}+$ $8 \mathrm{Ahp})$ or polypyrimidine (8Yhp $+\mathrm{RC}+8 \mathrm{Yhp})$ sequences and consisted of an 8 base pair (bp) stem associated with a 
A
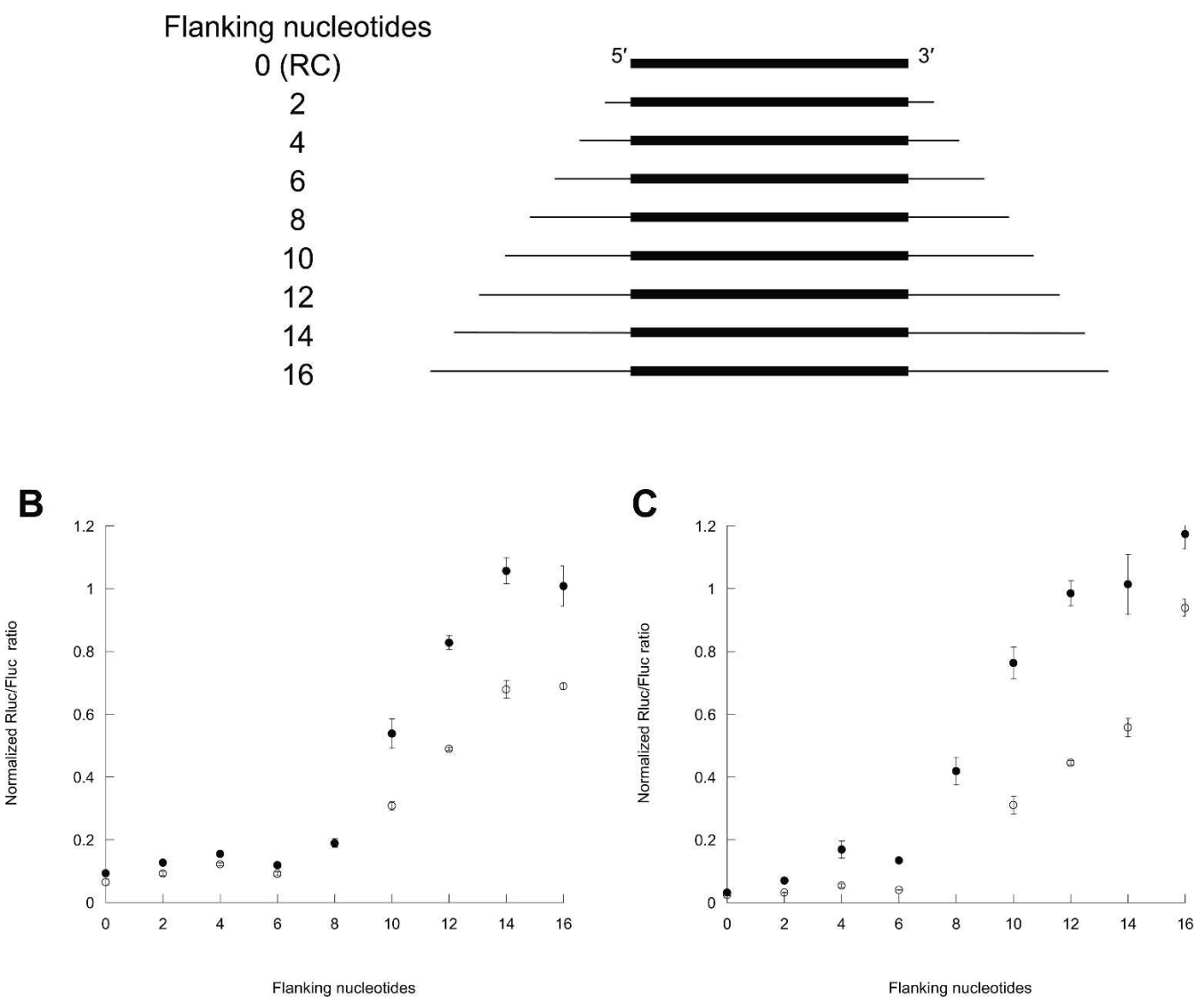

FIGURE 2. Overall length of $2^{\prime}$-O-methylated miRNA inhibitors affects functionality. $(A)$ Schematic showing 2'-O-methyl-modified inhibitors with central region reverse complement to mature (dark bar) and flanking regions (reverse complement to pri-miRNA) with lengths varying from 0 to $16 \mathrm{nt}$ on each side. Inhibitors targeting let-7c $(B)$ and miR-21 $(C)$ were assayed by cotransfecting into HeLa cells at 25 (open circles) and $50 \mathrm{nM}$ (filled circles) concentrations. The dual luciferase ratio was measured $48 \mathrm{~h}$ post-transfection to determine the effectiveness of each design.

tetranucleotide loop (Fig. 4). A direct comparison between the hairpin-containing inhibitors and single-stranded molecules comprising (1) an RC region alone, or (2) an RC region flanked by 8 -nt sequences, further supported the argument that secondary structure is a major positive contributor to inhibitor function. At subnanomolar concentrations $(0.78 \mathrm{nM})$ none of the single-stranded inhibitors of any configuration $(\mathrm{RC}, 8 \mathrm{~A}+\mathrm{RC}+8 \mathrm{~A}$, or $8 \mathrm{Y}+\mathrm{RC}+$ $8 \mathrm{Y}$ ) exhibited activity (Fig. $4 \mathrm{~A}, \mathrm{~B}$ ). In contrast, conversion of the single-stranded sequences into hairpins increased potency by $2-5$-fold with hairpins carrying polypyrimidine tracts exhibiting the greatest level of relative improvement.

The enhanced functionality brought about by incorporation of hairpin structures might be mimicked by the addition of double-stranded (ds) structures adjacent to the RC core. To test this hypothesis, two additional oligonucleotides were hybridized to the $5^{\prime}$ and $3^{\prime}$ flanking regions of poorly $(16 \mathrm{Y}+\mathrm{RC}+16 \mathrm{Y})$ and moderately $(16 \mathrm{~A}+$ $\mathrm{RC}+16 \mathrm{~A}$ ) functional single-stranded inhibitors. As predicted, conversion of the flanking regions from single- to double-stranded designs greatly enhanced overall functionality for both polypyrimidine and arbitrary flanking region inhibitors (Fig. 4).

Since (1) single-stranded 2'-O-methyl oligonucleotides may be sensitive to exonucleolytic degradation (Sproat et al. 1989; Cummins et al. 1995; Johnson et al. 2005), and (2) exonuclease activity generally proceeds in a $3^{\prime} \rightarrow$ $5^{\prime}$ direction, some asymmetry in the potency of hairpininhibitor designs might be expected when a hairpin is appended to the $5^{\prime}$ or $3^{\prime}$ end of the RC core. However, a test of inhibitors containing $5^{\prime}(16 \mathrm{hp}+\mathrm{RC}+16 \mathrm{~A})$ or $3^{\prime}$ $(16 \mathrm{~A}+\mathrm{RC}+16 \mathrm{hp})$ positioned hairpins failed to support this hypothesis, showing equal levels of functionality for both sets of molecules (Supplemental Fig. 4). In addition, incorporation of stabilized internucleotide linkages (e.g., 3' phosphorothioate modifications) also had little effect on overall potency (Supplemental Fig. 3), while substituting the 2'-O-methyl-modified stem with unmodified RNA had only a modest (negative) effect on inhibitor function (Supplemental Fig. 5). These results suggest that although 
A

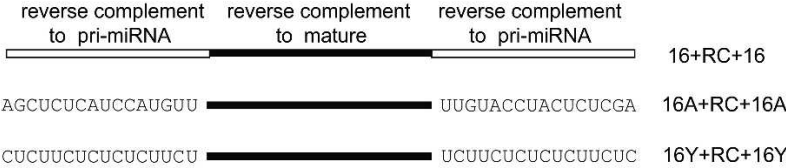

B

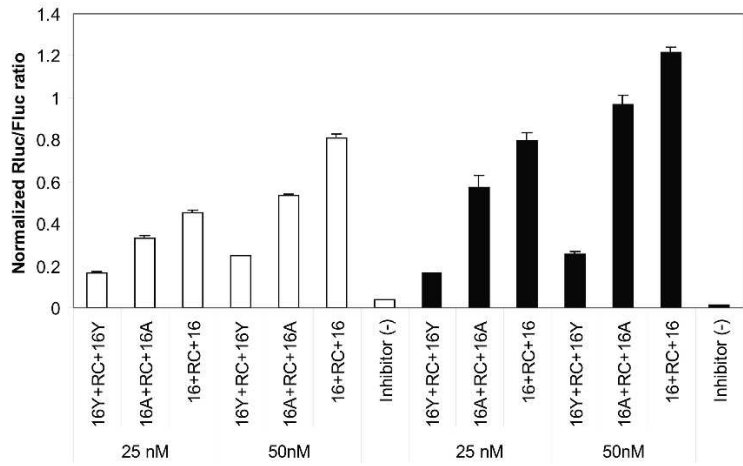

FIGURE 3. Inhibitor flanking sequence composition affects inhibitor functionality. (A) Schematic showing inhibitor designs containing various flanking compositions: unstructured poly-pyrimidine $(16 \mathrm{Y}+$ $\mathrm{RC}+16 \mathrm{Y})$, arbitrary $(16 \mathrm{~A}+\mathrm{RC}+16 \mathrm{~A})$, and reverse complement $(16+$ $\mathrm{RC}+16)$, around an antisense core. $(B)$ These inhibitor designs were tested for the ability to inhibit let-7c (white bars) or miR-21 (black bars) at concentrations of 25 and $50 \mathrm{nM}$. No-inhibitor control is shown (Inhibitor $[-]$ ).

the nuclease resistance of the flanking region could play a small role in improving inhibitor molecules, it does not appear to be a major determinant of potency for the hairpin-containing molecules. Instead, the increase in inhibitor functionality is potentially a result of additional binding affinity associated with sequence-independent or nonspecific nucleic acid recognition by RISC or RISCassociated factors.

\section{Double-stranded inhibitors enable combinatorial microRNA inhibition}

While miRNAs are believed to act as modulators of gene expression, there are few examples where overexpression or knockdown of a single miRNA results in a measurable phenotype (Esau et al. 2004; Poy et al. 2004; Yekta et al. 2004; Johnson et al. 2005; Lee et al. 2005; Lim et al. 2005; Chen et al. 2006; Schratt et al. 2006). This observation has led many in the field to speculate that miRNAs work in a combinatorial fashion and act in concert to target a single transcript (Krek et al. 2005). We compared the ability of single- and double-stranded inhibitors to simultaneously target multiple miRNAs to determine whether improved inhibitor design could address the issue of multi-miRNA knockdown. Specifically, three inhibitor designs (reverse complement to the mature miRNA [RC], $16+\mathrm{RC}+16$, and a hairpin-containing sequence) were tested for the ability to simultaneously silence all of the members of a polycistronic miRNA cluster encoding six separate miRNAs
(Fig. 5, miR-17-18a-19a-20-19b-92; Lagos-Quintana et al. 2001; Lau et al. 2001). Both of the single-stranded inhibitor pools ( $\mathrm{RC}$ and $16+\mathrm{RC}+16)$ failed to inhibit all the miRNAs in the cluster (particularly in the cases of miR-19a and miR-19b-1). In contrast, the hairpin-containing inhibitor pool efficiently repressed the function of all of the targeted miRNAs.

\section{DISCUSSION}

The structure-function studies described above identify two critical features associated with 2'-O-methyl-modified miRNA inhibitors. Extending the sequences beyond the boundaries of the RC core dramatically increases overall potency and identifies a minimal length necessary for potent sequence-specific miRNA inhibition. Interestingly, these studies also contribute to our understanding of the mode of action of 2'-O-methyl inhibitors. Prior experimentation with this class of inhibitors demonstrated that

A

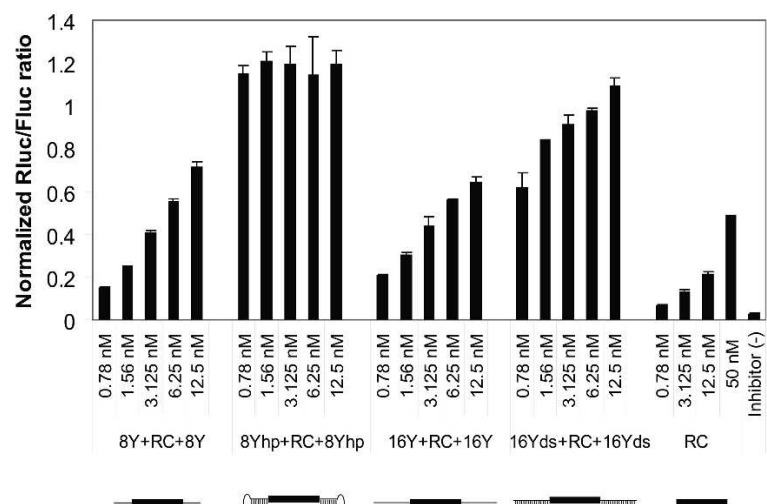

B

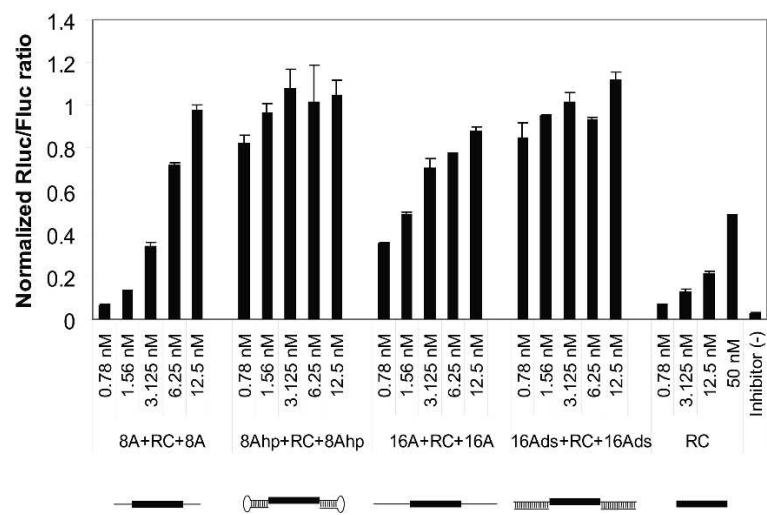

FIGURE 4. Inhibitor potency improves with the addition of doublestranded flanking regions. Inhibitors targeting miR-21 in HeLa cells were designed with flanking regions containing polypyrimidine $(A)$ and arbitrary $(B)$ sequences. Inhibitors containing 8 and $16 \mathrm{nt}$ singlestranded flanking regions $(8 \mathrm{Y}+\mathrm{RC}+8 \mathrm{Y}, 8 \mathrm{~A}+\mathrm{RC}+8 \mathrm{~A}, 16 \mathrm{Y}+\mathrm{RC}+$ $16 \mathrm{Y}, 16 \mathrm{~A}+\mathrm{RC}+16 \mathrm{~A})$ were compared to $8 \mathrm{bp}$ hairpin structures $(8 \mathrm{Yhp}+\mathrm{RC}+8 \mathrm{Yhp}, 8 \mathrm{Ahp}+\mathrm{RC}+8 \mathrm{Ahp})$ and $16 \mathrm{bp}$ double-stranded structures (16Yds + RC + 16Yds, 16Ads + RC + 16Ads). 
A

Chr 13 miR-17-92

17-5p $\quad 17-3 p \quad 18 a-5 p \quad 18 a-3 p$
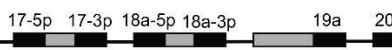

B

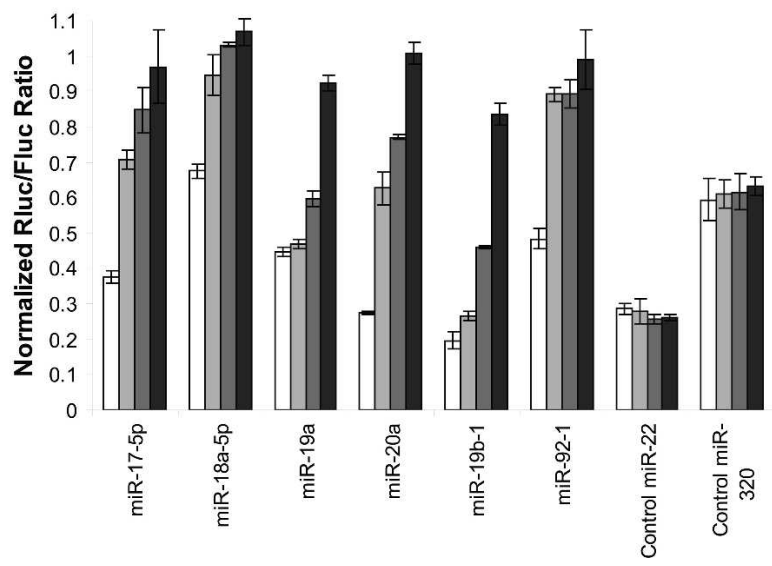

FIGURE 5. Combinatorial inhibition of microRNA function. (A) Diagram showing genomic organization of the mir-17-92 cluster on chromosome 13 (He et al. 2005). (B) Six miRNAs (mir-17-5p, mir18a-5p, mir-19a, mir-20, mir-19b-1, and mir-92-1) from the cluster were inhibited by pools of three different inhibitor designs (the reverse complement of the mature miRNA only [RC]; light gray bars), the reverse complement of the mature miRNA plus 16-nt flanking each side $(16+\mathrm{RC}+16$, dark gray bars), and the reverse complement of the mature miRNA flanked on both sides by hairpin sequences $(\mathrm{hp}+$ $\mathrm{RC}+\mathrm{hp}$, black bars). Values with no inhibitor are shown by white bars. All six inhibitors of each design were pooled for a $0.8 \mathrm{nM}$ total inhibitor concentration. These pools were cotransfected into HeLa cells with one of six reporter plasmids for each of the miRNAs or one of two control reporter plasmids for unrelated miRNAs (miR-22, miR-320) not included in the cluster.

they effectively inhibit miRISC particles in cytoplasmic extracts (Meister et al. 2004). Simultaneously, treatment of whole cells with fluorescently labeled molecules shows a predominant nuclear localization pattern (Y. Fedorov, pers. comm.). These two observations suggest that inhibitors may be acting at two separate levels in the RNAi pathway, inhibiting miRNA function in the nucleus by disrupting Drosha processing, and disrupting RISC-mediated targeting in the cytoplasm by acting as an alternative substrate (antisense mechanism). The observation that substitution of reverse complement flanking sequences with arbitrary sequences results in only a small decrease in overall functionality implies that the predominant site of action is in the cytoplasm and the mode of inhibition is possibly RISC driven.

Experiments that alter flanking sequence content and introduce regions of dsRNA have identified secondary structure as a second critical element in inhibitor function. One possible interpretation of this finding is that the presence of secondary structure stabilizes the inhibitors against extracellular or cytoplasmic nucleases; although data using asymmetrically appended hairpin inhibitor molecules and additional chemical stabilization of the flanking regions suggest this is not the case. An alternative hypothesis is that secondary structure enhances miRNARISC interactions with inhibitors.

Since secondary structure enhances inhibitor-miRNARISC association, analogous secondary structure in mRNAs may play a role in miRNA target and siRNA off-target selection. Current bioinformatic predictive algorithms show that there are many more seed complements (and thus potential miRNA target sites) than actual targeted genes. Thus, additional flanking elements (e.g., sequence motifs, secondary structures) could play critical roles in miRNA target site recognition. In the absence of other interactions, 6-7 bp in the seed region are insufficient to form a stable complex between the mature miRNA strand and the prospective mRNA target. Therefore, it is possible that dsRNA binding domains within Argonaute 2 or other RISC-associated proteins provide the necessary affinity to ensure stable miRNA-RISC-mRNA association.

Although miRNAs were identified over a decade ago, there have been few reports tying miRNA function to changes in gene expression and/or phenotypic changes. This fact speaks to the complexity of miRNA-mediated gene regulation and the need for effective tools to inhibit and mimic miRNA function. Because predicted target genes often contain target sites for multiple miRNAs, and clusters of miRNAs are coexpressed, it is possible that endogenous miRNAs act in combination to effect changes in target gene expression. If this is the case, tools that allow multi-miRNA knockdown will be essential for identification and validation of miRNA targets. As shown here, inhibition of multiple miRNAs by shorter, single-stranded inhibitors is limited. In contrast, incorporation of secondary structures into the flanking regions of miRNA inhibitors enhances miRNA inhibitor potency by several fold, effectively allowing as many as six miRNAs to be targeted simultaneously. As a result, these potent double-stranded miRNA inhibitors will enable more-detailed studies of the complex miRNA target network.

\section{MATERIALS AND METHODS}

\section{Plasmid reporters}

The dual-luciferase plasmid, psiCHECK-2 vector, containing both the synthetic firefly luciferase gene (hluct) and the synthetic Renilla luciferase gene (hRluc), each with its own promoter and poly(A)-addition sites, was obtained from Promega (Catalog No. C8021). miRNA target sequences were inserted between the XhoINot I restriction sites in the multiple cloning region in the $3^{\prime}$ UTR of the hRluc gene. Cleavage target sites are reverse complements of their respective predicted mature miRNAs (Sanger Institute miRBase::Sequences, http://microrna.sanger.ac.uk). Attenuation target sites contain a four-base insert between positions 8 and 9 of the mature miRNA and multiple attenuation target sites are composed of tandem repeats of the same insert. Insert sequences were 
ordered from Sigma-Genosys (sequences shown in Supplemental Table 1) to make an insert compatible with the restriction sites.

\section{siRNAs and inhibitors}

The hRluc siRNA pool is a SMARTselection designed siRNA pool and the control siRNA is directed against PPIB (Catalog No. D-004606-04) from Thermo Fisher Scientific, Dharmacon Products. Inhibitors targeting miR-21, let-7c, miR-17-5p, miR-18a-5p, miR-19a, miR-20, miR-19b-1, and miR-92-1 were synthesized by Thermo Fisher Scientific, Dharmacon Products and were fully 2'-O-methylated unless otherwise indicated. Sequences for all inhibitors are reported in Supplemental Table 2.

\section{Transfection}

HeLa cells, which express both miR-21 and let-7 at high levels, were obtained from ATCC and cultured under recommended media conditions. HeLa cells were plated in serum-containing media without antibiotics in 96-well plates $\left(1 \times 10^{4}\right.$ cells/well $)$ 18-24 h before transfection. Cells were transfected with $0.8-$ $50 \mathrm{nM}$ inhibitor and $100 \mathrm{ng} /$ well of the reporter plasmid using $0.3 \mu \mathrm{L} /$ well Lipofectamine 2000 (Invitrogen). Cells were grown at $37^{\circ} \mathrm{C}$ and harvested at $24-48 \mathrm{~h}$ after transfection for luciferase, branched DNA, and viability assays.

\section{Measuring luciferase activity, cell viability, and mRNA levels}

Firefly and Renilla luciferase activities were measured using the Dual-Glo Luciferase Assay System (Promega, Catalog No. E2980) according to manufacturer's instructions with slight modifications. When lysing cells, growth media were aspirated from the cells prior to adding $50 \mu \mathrm{L}$ of firefly luciferase substrate and $50 \mu \mathrm{L}$ Renilla luciferase substrate.

Cell viability was determined on a duplicate plate using the alamarBlue assay (BioSource Int., Inc.). Cell viabilities for control and experimentally treated cells were always within $15 \%$.

For experiments requiring the quantitative determination of mRNA, total RNA was extracted from transfected cells using the SV 96 Total RNA Isolation System (Promega, Catalog No. Z3505). The entire extract was then used for the determination of mRNA levels by branched DNA assay (QuantiGene Screen Kit, Catalog No. QG-000-050, Panomics) (Collins et al. 1997). Branched DNA probes for hRluc and hluc+ were designed by Panomics.

The luciferase, alamarBlue, and bDNA assays were all read with a Wallac Victor ${ }^{2} 1420$ multilabel counter (PerkinElmer) using programs as recommended by the manufacturers.

\section{Experimental design and data analysis}

All treatments were performed in triplicate. In addition, each experimental treatment with a reporter plasmid was duplicated with the psiCHECK-2 control plasmid (no insert). To account for nonspecific effects on reporter plasmids, experimental results are expressed as a normalized ratio (Rluc/Fluc) norm: the ratio of Renilla luciferase expression to firefly luciferase expression for a given miRNA reporter plasmid (Rluc/Fluc) miRNA divided by the (Rluc/Fluc) control ratio for the identically treated psiCHECK-2 reporter plasmid (no insert). The maximum values obtained from the reporter plasmid vary due to sequence; ideally, values $\sim 1$ indicate low miRNA function, while values close to zero indicate high miRNA function. Data are reported as the average of the triplicates and the error bars are the standard deviation of the three (Rluc/Fluc) miRNA ratios from the experimental treatment, scaled by the normalizing factor (the average of [Rluc/Fluc $]_{\text {control }}$ ). We recognize that ratios do not follow a normal distribution, but we believe that the standard deviation values give an accurate measure of the variability of the data.

In cases where values between different miRNA reporter plasmids are compared (Figs. 1, 5), the maximum normalized (Rluc/ Fluc) norm ratio was used as an additional scaling factor so that all reporters have a maximum of $\sim 1$. The additional scaling was performed for ease of comparison and does not affect the results.

\section{SUPPLEMENTAL DATA}

Supplemental Materials are available at http://www.dharmacon. com/tech/publications/.

\section{ACKNOWLEDGMENTS}

We acknowledge the R\&D department for critical discussions and the Production Team at Thermo Fisher Scientific, Dharmacon Products for oligonucleotide synthesis.

Received December 24, 2006; accepted February 19, 2007.

\section{REFERENCES}

Bino, J., Enright, A.J., Aravin, A., Tuschl, T., Sander, C., and Marks, D.S. 2004. Human microRNA targets. PLoS Biol. 2: 1862-1879.

Chen, J.F., Mandel, E.M., Thomson, J.M., Wu, Q., Callis, T.E., Hammond, S.M., Conlon, F.L., and Wang, D.Z. 2006. The role of microRNA-1 and microRNA-133 in skeletal muscle proliferation and differentiation. Nat. Genet. 38: 228-233.

Collins, M.L., Irvine, B., Tyner, D., Fine, E., Zayati, C., Chang, C., Horn, T., Ahle, D., Detmer, J., Shen, L.P., et al. 1997. A branched DNA signal amplification assay for quantification of nucleic acid targets below 100 molecules/ml. Nucleic Acids Res. 25: 2979-2984.

Cummins, L., Owens, S., Risen, L., Lesnik, E., Freier, S., McGee, D., Guinosso, C., and Cook, P. 1995. Characterization of fully 2'modified oligoribonucleotide hetero- and homoduplex hybridization and nuclease sensitivity. Nucleic Acids Res. 23: 2019-2024.

Davis, S., Lollo, B., Freier, S., and Esau, C. 2006. Improved targeting of miRNA with antisense oligonucleotides. Nucleic Acids Res. 34: 2294-2304.

Enright, A., John, B., Gaul, U., Tuschl, T., Sander, C., and Marks, D. 2003. MicroRNA targets in Drosophila. Genome Biol. 5: R1.

Esau, C., Kang, X., Peralta, E., Hanson, E., Marcusson, E.G., Ravichandran, L.V., Sun, Y., Koo, S., Perera, R.J., Jain, R., et al. 2004. MicroRNA-143 regulates adipocyte differentiation. J. Biol. Chem. 279: 52361-52365.

Giraldez, A.J., Mishima, Y., Rihel, J., Grocock, R.J., VanDongen, S., Inoue, K., Enright, A.J., and Schier, A.F. 2006. Zebrafish MiR-430 promotes deadenylation and clearance of maternal mRNAs. Science 312: 76-79.

Gregory, R.I., Chendrimada, T.P., and Shiekhattar, R. 2006. MicroRNA biogenesis: Isolation and characterization of the microprocessor complex. Methods Mol. Biol. 342: 33-47.

He, L., Thomson, J.M., Hemann, M.T., Hernando-Monge, E., Mu, D., Goodson, S., Powers, S., Cordon-Cardo, C., Lowe, S.W., Hannon, G.J., et al. 2005. A microRNA polycistron as a potential human oncogene. Nat. Lett. 435: 828-833. 
Hutvagner, G., Simard, M., Mello, C., and Zamore, P. 2004. Sequencespecific inhibition of small RNA function. PLoS Biol. 2: E98.

Johnson, S., Grosshans, H., Shingara, J., Byrom, M., Jarvis, R., Cheng, A., Labourier, E., Reinert, K., Brown, D., and Slack, F. 2005. RAS is regulated by the let-7 MicroRNA family. Cell 120: 635-647.

Kim, V.N. 2005. MicroRNA biogenesis: Coordinated cropping and dicing. Nat. Rev. Mol. Cell Biol. 6: 376-385.

Kiriakidou, M., Nelson, P.T., Kouranov, A., Fitziev, P., Bouyioukos, C., Mourelatos, Z., and Hatzigeorgiou, A. 2004. A combined computational-experimental approach predicts human microRNA targets. Genes \& Dev. 18: 1165-1178.

Krek, A., Grun, D., Poy, M., Wolf, R., Rosenberg, L., Epstein, E., MacMenamin, P., da Piedade, I., Gunsalus, K., Stoffel, M., et al. 2005. Combinatorial microRNA target predictions. Nat. Genet. 37: 495-500.

Krutzfeldt, J., Rajewsky, N., Braich, R., Rajeev, K., Tuschl, T., Manoharan, M., and Stoffel, M. 2005. Silencing of microRNAs in vivo with "antagomirs." Nature 438: 685-695.

Lagos-Quintana, M., Rauhut, R., Lendeckel, W., and Tuschl, T. 2001. Identification of novel genes coding for small expressed RNAs. Science 294: 853-858.

Lau, N.C., Lim, L.P., Weinstein, E.G., and Bartel, D.P. 2001. An abundant class of tiny RNAs with probable regulatory roles in Caenorhabditis elegans. Science 294: 858-862.

Lee, Y.S., Kim, H.K., Chung, S., Kim, K.S., and Dutta, A. 2005. Depletion of human micro-RNA miR-125b reveals that it is critical for the proliferation of differentiated cells but not for the downregulation of putative targets during differentiation. J. Biol. Chem. 280: 16635-16641.

Lewis, B., Burge, C., and Bartel, D. 2005. Conserved seed pairing, often flanked by adenosines, indicates that thousands of human genes are microRNA targets. Cell 120: 15-20.

Lim, L.P., Glasner, M.E., Yekta, S., Burge, C.B., and Bartel, D.P. 2003. Vertebrate microRNA genes. Science 299: 1540.

Lim, L., Lau, N., Garrett-Engele, P., Grimson, A., Schelter, J., Castle, J., Bartel, D., Linsley, P., and Johnson, J. 2005. Microarray analysis shows that some microRNAs downregulate large numbers of target mRNAs. Nature 433: 769-773.
Liu, J., Valencia-Sanchez, A.M., Hannon, G.J., and Parker, R. 2005. MicroRNA-dependent localization of targeted mRNAs to mammalian P-bodies. Nat. Cell Biol. 7: 719-723.

Meister, G., Landthaler, M., Dorsett, Y., and Tuschl, T. 2004. Sequence-specific inhibition of microRNA- and siRNA-induced RNA silencing. RNA 10: 544-550.

Orom, U.A., Kauppinen, S., and Lund, A.H. 2006. LNA-modified oligonucleotides mediate specific inhibition of microRNA function. Gene 372: 137-141.

Pillai, R.S., Bhattacharyya, S.N., Artus, C.G., Zoller, T., Cougot, N., Basyuk, E., Bertrand, E., and Filipowicz, W. 2005. Inhibition of translational initiation by let-7 MicroRNA in human cells. Science 309: 1573-1576.

Poy, M., Eliasson, L., Krutzfeldt, J., Kuwajima, S., Ma, X., Macdonald, P., Pfeffer, S., Tuschl, T., Rajewsky, N., Rorsman, P., et al. 2004. A pancreatic islet-specific microRNA regulates insulin secretion. Nature 432: 226-230.

Rhoades, M.W., Reinhart, B.J., Lim, L.P., Burge, C.B., Bartel, B., and Bartel, D.P. 2002. Prediction of plant microRNA targets. Cell 110: 513-520.

Schratt, G.M., Tuebing, F., Nigh, E.A., Kane, C.G., Sabatini, M.E., Kiebler, M., and Greenberg, M.E. 2006. A brain-specific microRNA regulates dendritic spine development. Nature 439: 283-289.

Sen, G.L. and Blau, H.M. 2005. Argonaute 2/RISC resides in sites of mammalian mRNA decay known as cytoplasmic bodies. Nat. Cell Biol. 7: 633-636.

Sethupathy, P., Corda, B., and Hatzigeorgiou, A.G. 2006. TarBase: A comprehensive database of experimentally supported animal microRNA targets. RNA 12: 192-197.

Sproat, B.S., Lamond, A.I., Beijer, B., Neuner, P., and Ryder, U. 1989. Highly efficient chemical synthesis of 2'-O-methyloligoribonucleotides and tetrabiotinylated derivatives; novel probes that are resistant to degradation by RNA or DNA specific nucleases. Nucleic Acids Res. 17: 3373-3386.

Yekta, S., Shih, I.H., and Bartel, D.P. 2004. MicroRNA-directed cleavage of HOXB8 mRNA. Science 304: 594-596. 

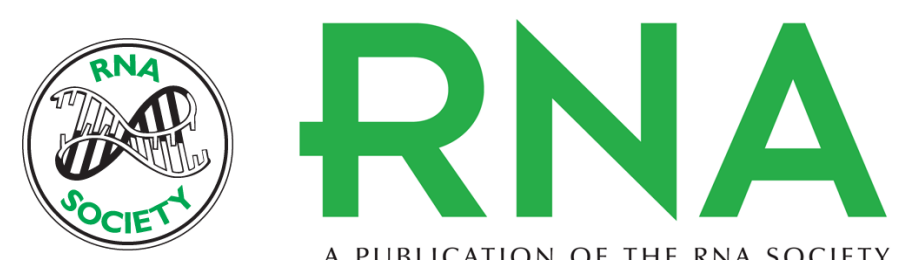

A PUBLICATION OF THE RNA SOCIETY

\section{Double-stranded regions are essential design components of potent inhibitors of RISC function}

Annaleen Vermeulen, Barbara Robertson, Andrew B. Dalby, et al.

RNA 2007 13: 723-730 originally published online March 30, 2007

Access the most recent version at doi:10.1261/rna.448107

\section{References This article cites 33 articles, 10 of which can be accessed free at: http://rnajournal.cshlp.org/content/13/5/723.full.html\#ref-list-1}

Open Access Freely available online through the RNA Open Access option.

License Freely available online through the open access option.

Email Alerting Receive free email alerts when new articles cite this article - sign up in the box at the Service top right corner of the article or click here.

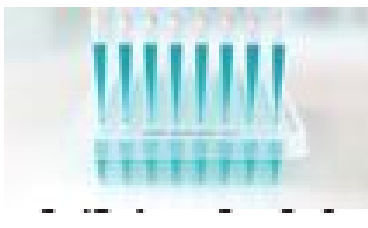

